\title{
Classification of Breast Masses based on Cognitive Resonance
}

\author{
Amir Tahmasbi, Fatemeh Saki, Abdollah Amirkhani, Seyed Mohammad Seyedzade, and Shariar B. \\ Shokouhi
}

\begin{abstract}
In this paper, a novel approach has been proposed for mass diagnosis in mammography images. The objective is developing a trustable mammography Computer Aided-Diagnosis (CADx) system utilizing a new cognitive classifier. The input Region of Interest (ROI) is subjected to some preprocessing stages; then, a group of features describing the shape, margin and density characteristics of masses have been extracted. The proposed features are consistent with the evaluations that an expert radiologist takes into account in diagnosis process. The most effective features are selected in the feature selection stage and mapped from the set of real numbers to a set of linguistic terms. The proposed classifier primes a knowledge-base which is developed according to a mammography expert; its rules have been written using a special kind of linguistics and grammar formalism. The semantic comparison of features of the image to the expectations of the knowledge base, called cognitive resonance, leads to the final assessment. Since the output of this system comes with reason, the system is trustable. The best achieved Accuracy and False Positive Rate (FPR) are $87.93 \%$ and $10.52 \%$, respectively. More numerical results are reported in the paper.
\end{abstract}

Index Terms-Cognitive pattern recognition, cognitive resonance, computer aided diagnosis, mammography.

\section{INTRODUCTION}

Breast cancer has always been one of the most perilous diseases among women [1]. Despite technology advances in the fields of mammography [2]-[6], thermography [7], optical tomography [8] and other anticancer methodologies in the last two decades, breast cancer is still a prominent problem. Early detection of breast cancer increases the survival rate as well as the treatment options [2]. Mammography has been one of the most reliable methods for early detection and diagnosis of breast cancers [9]. Some of the important signs of breast cancer that radiologists look for are clusters of micro-calcifications, masses, and architectural distortions [2], [3]. A mass is defined as a space-occupying lesion seen in at least two different projections [2]. Current research is directed toward the development of a CADx system for mass diagnosis in mammography images based on a cognitive approach.

The traditional approach to automated analysis of medical data is mostly based on statistical pattern recognition [10].

Manuscript received April 9, 2012; revised May 10, 2012.

A. Tahmasbi is with the Department of Electrical Engineering, University of Texas at Dallas, Texas, U.S. (e-mail: a.tahmasbi@utdallas.edu).

F. Saki and S. B. Shokouhi are with the Department of Electrical Engineering, Iran University of Science and Technology (IUST), Tehran, Iran (e-mail: f_saki@elec.iust.ac.ir, bshokouhi@iust.ac.ir).
Unfortunately, such systems do not focus on the context of the features [10] while a CADx system may need something more than traditional feature extraction algorithms. To develop a trustable system we need to use the features which are extracted in an in-depth analysis process.

In addition, either Artificial Neural Network (ANN) or statistical algorithms have often been used as classifiers in medical diagnosis systems [2]. Although capable of achieving an optimal performance, developing an ANN-based classifier would be rather time-consuming since it may take a few hundreds to thousands of runs before figuring out the appropriate parameters [7]. Moreover, statistical methods are difficult to develop, and they often work under the assumption that the underlying data are normally distributed [7]. The most important disadvantage of all of these methods is that they do not provide any explanations for their computations and reasoning. Consequently, there is no way to validate the operation of system, and the output is difficult to trust because it comes without reason.

On the other hand, it might be useful to make semantic content analysis leading to automatic understanding of medical data instead of their simple processing and analysis. In this paper, an expert system has been developed which utilizes in-depth analyzed features and a cognitive classifier in order to diagnose breast masses. In fact, a number of features have been extracted; then, some of them are selected and mapped from the set of real numbers to a set of linguistic terms. Moreover, a knowledge base has been provided based on the knowledge of a mammography expert which contains some linguistic rules. The linguistic rules are written according to a special kind of grammar. The semantic comparison of features of real image with the expectations of knowledge base, called cognitive resonance [11], leads to the final assessment. This kind of classification is called Cognitive Classification. Fortunately, the reasoning of the proposed system is very close to a mammography expert's inferences and the system is trustable.

Cognitive classification has been used in several diagnostic support systems (DSS) and yielded interesting results. For example, it has been utilized in the diagnosis of spinal cord [11] and foot bone image analysis [12].

In this paper, the Mammographic Image Analysis Society (MIAS) database has been utilized to provide the mammography images [13]. It contains 322 digital mammograms which belong to right and left breasts of 161 different women. Each mammogram has been digitized with a resolution equal to 200 micron pixel edge and stored in a $1024 \times 1024$ pixels digital image. The MIAS database includes 209 normal breasts, 67 ROIs with benign lesions and 
54 ROIs with malignant lesions [13]. Note that some breasts may have more than one lesion.

In the next section, the proposed algorithm is explained in detail. The experiments and results are represented in Section III. Eventually, in the last section, a conclusion has been made.

\section{Methodology}

Fig. 1 illustrates the flowchart of the proposed cognitive approach. The input data is an ROI which is a suspected part of a mammogram and contains only one mass. In this research, the ROIs are assumed to have equal width and length. Each stage of proposed approach is discussed in detail as follows.

\section{A. Preprocessing and Segmentation}

In this paper, manual segmentation has been utilized. In fact, each ROI is segmented by two different expert radiologists and the final boundary of mass was calculated using radial averaging.

Table I shows the employed features. Each of the proposed features needs some special kind of preprocessing steps. For instance, circularity, contrast, and average gray level measures need segmentation [9]. Zernike moments [3], [4], [14] need segmentation, co-scaling using NRL vector and translation. NRL features also need segmentation [5], [15]. Eventually, SpI feature [16] needs segmentation, co-scaling using NRL vector, translation and histogram equalization. Fig. 2 illustrates an ROI in different steps of the preprocessing stage. After the preprocessing stage, the ROIs are ready to be applied to the feature extraction stage.

\section{B. Feature Extraction and Selection}

According to Breast Imaging-Reporting and Data System (BI-RADS), expert radiologists focus on three different types of features while visually searching the mammograms [2]. These three types of features are shape, margin, and density. Mass shape can be round, oval, lobulated or irregular. Mass margin can be circumscribed, microlobulated, indistinct, or spiculated. Density features represent relative mass density with respect to normal fibroglandular breast tissue and include high density, isodense, low density, and radiolucent masses [2]. The implied features are exactly similar to those ones which an expert radiologist takes into account in the diagnosis process. Hence, if one can extract them by an appropriate method, the resulting CADx system may achieve better performances than traditional approaches. The proposed eleven features tabulated in Table I can cover all the BI-RADS categories. Circularity [9] and Zernike moments [3], [4], [14] are proper descriptors of mass shape. The NRL derivatives [5], [15] and SpI [16] are appropriate descriptors of mass margin. Moreover, contrast and average gray level [9] are suitable descriptors of mass density. Note that the order and iteration of utilized Zernike moments have been chosen so that they can represent a good descrimination between benign and malignant masses. The features are applied to the feature selection stage and the most effective ones are selected. Effective features are the ones that not only are good descriptors of mass characteristics, but also have little overlap with other features. Besides, those features that have high computational complexity and represent unacceptable performance have been neglected in this stage. The final features are $\mathrm{C}, Z_{4}^{2}, Z_{6}^{0}$, NRLs, NRLzcc, NRLen, and Contrast.

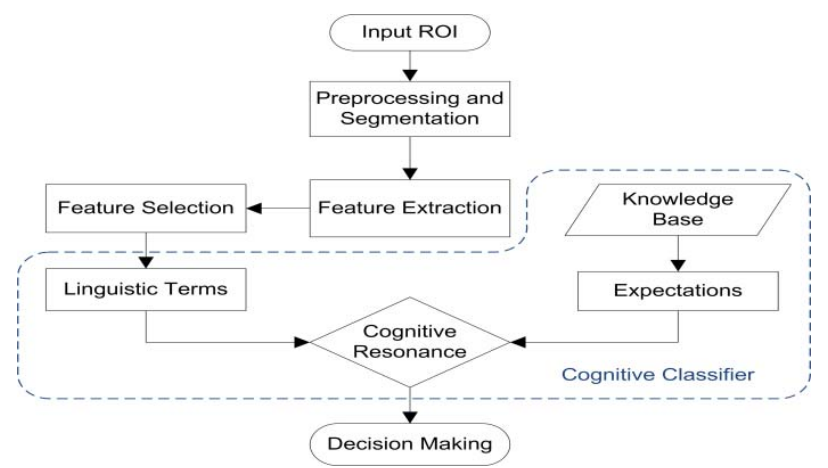

Fig. 1. The block diagram of proposed cognitive approach for mass diagnosis in mammography images

TABLE I: THE UTILIZED FEATURES

\begin{tabular}{lll}
\hline \hline Category & Feature name & Explanation \\
\hline \multirow{4}{*}{ Shape } & $\mathrm{C}$ & Circularity \\
& $Z_{4}^{2}$ & \\
& $Z_{6}^{0}$ & Zernike moments \\
& $Z_{9}^{7}$ & \\
\hline \multirow{5}{*}{ Margin } & NRLs & NRL standard deviation \\
& NRLzcc & NRL zero crossing count \\
& NRLar & NRL area ratio \\
& NRLen & NRL entropy \\
& SpI & Spiculation Index \\
\hline \multirow{2}{*}{ Density } & AGL & Average gray level \\
& Con & Contrast \\
\hline \hline
\end{tabular}

\section{Cognitive Classification}

In this stage, the selected features are mapped from the set of real numbers to a set of linguistic terms which are developed using a special kind of grammar; the proposed grammar is consistent with the semantic information used by an expert radiologist in a diagnosis process. Moreover, the set of linguistic terms includes some non-terminal linguistic variables which are representatives of various BI-RADS categories. For instance, semantic analysis may reveal that suspected mass belongs to lobulated category from the viewpoint of shape characteristics.

For cognitive analysis of the extracted features, a context-free grammar was prepared, which allows for the identification of mass types according to BI-RADS and drawing of diagnostic conclusion concerning the malignancy of masses [11]. This context-free grammar is given as follows [12].

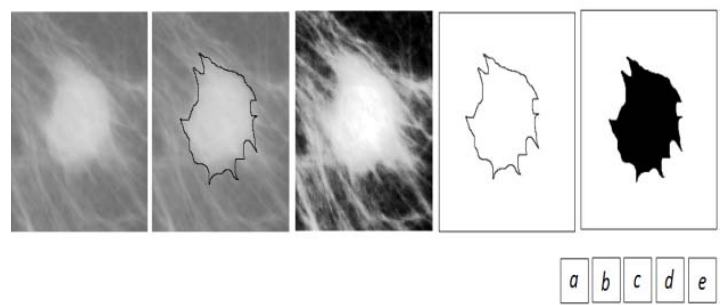

Fig. 2. a) Input ROI containing only one mass. b) Manual segmentation of mass by an expert radiologist. c) Input ROI after histogram equalization. d) Mass boundary after segmentation in binary format. e) Mass shape in binary format 


$$
G_{m}=\left(\Sigma_{N}, \Sigma_{T}, P, S T\right)
$$

where $\Sigma_{N}$ is the set of non-terminal symbols, $\Sigma_{T}$ is the set of terminal symbols, $P$ is the set of productions, and $S T$ is the starting symbol of grammar. The set of non-terminal symbols are given as follows.

$$
\begin{aligned}
& \sum_{N}=\{\text { FINAL_ASSESS , BENIGN , MALIGNANT , } \\
& \text { ROUD , OVAL , LOBUL , IRREG ,CIRC , } \\
& \text { MICROL , INDIST , SPIC , HDENS , } \\
& \text { ISODENS , LDENS , } C 1, C 2, C 3, C 4, A 1, A 2 \text {, } \\
& B 1, B 2, B 3, B 4, B 5, B 6, D 1, D 2, D 3, D 4 \text {, } \\
& E 1, E 2, F 1, F 2, G 1, G 2, G 3\}
\end{aligned}
$$

And here is the set of terminal symbols.

$$
\begin{array}{r}
\Sigma_{T}=\{c 1, c 2, c 3, c 4, a 1, a 2, b 1, b 2, b 3, b 4, b 5, b 6, \\
d 1, d 2, d 3, d 4, e 1, e 2, f 1, f 2, g 1, g 2, g 3\}
\end{array}
$$

where the members of $\Sigma_{T}$ are the selected features in the linguistic space. The mapping functions from the set of real numbers to a set of linguistics for each feature are given as follows. Note that the mapping intervals have been found by a close investigation in the features' behaviors.

$$
\begin{aligned}
& c 1=\{C \mid C \in[12.57,24)\} \\
& c 2=\{C \mid C \in[24,28)\} \\
& c 3=\{C \mid C \in[28,34)\} \\
& c 4=\{C \mid C \in[34,100]\} \\
& b 1=\left\{Z_{6}^{0} \mid Z_{6}^{0} \in[0,0.03]\right\} \\
& b 2=\left\{Z_{6}^{0} \mid Z_{6}^{0} \in(0.03,0.12)\right\} \\
& b 3=\left\{Z_{6}^{0} \mid Z_{6}^{0} \in[0.12,0.2]\right\} \\
& b 4=\left\{Z_{6}^{0} \mid Z_{6}^{0} \in(0.2,0.26)\right\} \\
& b 5=\left\{Z_{6}^{0} \mid Z_{6}^{0} \in[0.26,0.33]\right\} \\
& b 6=\left\{Z_{6}^{0} \mid Z_{6}^{0} \in(0.33,1]\right\} \\
& a 1=\left\{Z_{4}^{2} \mid Z_{4}^{2} \in[0,0.2)\right\} \\
& a 2=\left\{Z_{4}^{2} \mid Z_{4}^{2} \in[0.2,1)\right\} \\
& d 1=\{Z c c \mid Z c c \in[0,5]\} \\
& d 2=\{Z c c \mid Z c c \in(5,9)\} \\
& d 3=\{Z c c \mid Z c c \in[9,12)\} \\
& d 4=\{Z c c \mid Z c c \in[12,100]\} \\
& e 1=\{\text { en } \mid \text { en } \in[6,6.1)\} \\
& e 2=\{e n \mid \text { en } \in[6.1,7]\}
\end{aligned}
$$

$$
\begin{aligned}
& g 1=\{\text { Con } \mid \text { Con } \in[0,15)\} \\
& g 2=\{\text { Con } \mid \text { Con } \in[15,100]\} \\
& g 3=\{\text { Con } \mid \text { Con } \in[-100,0)\}
\end{aligned}
$$

Moreover, $S P=$ FINAL_ASSESS is the starting point of the grammar. And eventually, the production set, $P$, which contains some linguistic rules, is tabulated in Table II. Note that the production set equals to the utilized knowledge base.

It is important to say that the simplicity of these grammar are mainly from the great generation capacity of context-free grammars, which are understood as the possibilities to describe complex shapes by means of small number of introductory rules that are grammar productions [11], [12].

Classification based on cognitive resonance is simple and low cost from the view point of computational complexity. In fact, parser begins from the starting point $(S P)$ of the

\begin{tabular}{|c|c|c|c|c|c|c|c|}
\hline Name & Explanation & $\begin{array}{l}\text { No. of } \\
\text { rules }\end{array}$ & $\begin{array}{l}\text { No. of test } \\
\text { patterns }\end{array}$ & $\begin{array}{l}\text { No. of misclassified } \\
\text { ROIs }\end{array}$ & FPR & FNR & Accuracy \\
\hline S0 & ideal & 38 & 58 & 7 & $10.52 \%$ & $15.1 \%$ & $87.94 \%$ \\
\hline S1 & $+10 \%$ random noise & 38 & 58 & 7 & $10.52 \%$ & $15.2 \%$ & $87.93 \%$ \\
\hline S2 & $+20 \%$ random noise & 38 & 58 & 8 & $13.15 \%$ & $15.2 \%$ & $86.21 \%$ \\
\hline S3 & $+30 \%$ random noise & 38 & 58 & 9 & $15.78 \%$ & $15.2 \%$ & $84.48 \%$ \\
\hline S4 & $+40 \%$ random noise & 38 & 58 & 11 & $21.42 \%$ & $19.9 \%$ & $81.03 \%$ \\
\hline S5 & $+50 \%$ random noise & 38 & 58 & 14 & $23.68 \%$ & $25.1 \%$ & $75.86 \%$ \\
\hline
\end{tabular}
grammar and parses the whole grammar to generate a discrete linguistic output.

\section{EXPERIMENTS AND RESULTS}

The prominent benchmarks of a CADx system are True Positive Rate (TPR), FPR, Accuracy, Receiver Operational Characteristics curve (ROC), and the area under it ( $A z)$ [2], [3], [9]. Moreover, the most common way of reporting the accuracy of a binary prediction is using the true (or false) positives and true (or false) negatives separately. This recognizes that a false negative prediction may have different consequences from the false positive one. In our research, a false-positive answer is less important than a false-negative answer. A plot of Sensitivity (TPR) vs. 1-Specificity (FPR) with changing the decision threshold is called ROC curve. The area under the ROC curve, $A z$, shows how successful the classification is [9].

Unfortunately, the decision making in this research is discrete and binary, so we cannot plot the ROC curve. However, the FNR, Accuracy and FPR measures for the proposed system are calculated and tabulated in Table III. The first row of the table belongs to the system with an ideal mapping stage. The other rows belong to those systems in which a White Gaussian Noise (WGN) applied to the mapping intervals in, i.e. rows (4)-(10). In fact, if $M$ is the mapping interval, then for $\mathrm{S} 1-\mathrm{S} 5$ the mapping intervals will be $M+N(0, \sigma)$ where $\sigma=r \times M$ and $r$ is one of the percentages given in the Table III. According to performance parameters reported in the table, it can be inferred that the system is tolerable to changes in the mapping intervals. 
Table II: The Production Set of Proposed Cognitive ApProach

\begin{tabular}{|c|c|c|}
\hline & & \\
\hline Lesion & Rule No. & Production set \\
\hline Benign & 1 & FINAL_ASSESS $\rightarrow$ BENIGN \\
\hline & 2 & $\begin{aligned} \text { BENIGN } \rightarrow & \text { ROUND. HDENS. CIRC | ROUND. HDENS. MICROL | ROUND. ISODENS. CIRC } \mid \\
& \text { ROUND. ISODENS. MICROL | OVAL. HDENS. CIRC | OVAL. HDENS. MICROL } \mid \\
& \text { OVAL. ISODENS. CIRC | OVAL. ISODENS. MICROL }\end{aligned}$ \\
\hline Malignant & 3 & FINAL ASSESS $\rightarrow$ MALIGNANT \\
\hline & 4 & $\begin{aligned} \text { MALIGNANT } \rightarrow & \text { IRREG. HDENS. SPIC | IRREG. HDENS. INDIST | IRREG. ISODENS. SPIC | } \\
& \text { IRREG. ISODENS. INDIST | LOBUL. HDENS. SPIC | LOBUL. HDENS. INDIST } \\
& \mid \text { LOBUL. ISODENS. SPIC | LOBUL. ISODENS. INDIST }\end{aligned}$ \\
\hline & 5 & ROUND $\rightarrow$ C3. B5 $\mid$ C1. A1 $\mid \mathrm{C} 2$. B5 \\
\hline Shape & 6 & $\mathrm{OVAL} \rightarrow \mathrm{C} 1 . \mathrm{A} 2|\mathrm{C} 3 . \mathrm{B} 2| \mathrm{C} 2 . \mathrm{B} 6|\mathrm{C} 2 . \mathrm{B} 4| \mathrm{C} 2 . \mathrm{B} 3|\mathrm{C} 2 . \mathrm{B} 2| \mathrm{C} 2 . \mathrm{B} 1$ \\
\hline features & 7 & LOBUL $\rightarrow$ C3. B4 \\
\hline & 8 & IRREG $\rightarrow \mathrm{C} 4|\mathrm{C} 3 . \mathrm{B} 1| \mathrm{C} 3 . \mathrm{B} 3$ \\
\hline & 9 & $\mathrm{CIRC} \rightarrow \mathrm{D} 1 \mid \mathrm{D} 3 . \mathrm{E} 1 . \mathrm{F} 1$ \\
\hline Margin & 10 & MICROL $\rightarrow$ D2 \\
\hline features & 11 & INDIST $\rightarrow$ D3. E2. F2 \\
\hline & 12 & $\mathrm{SPIC} \rightarrow \mathrm{D} 4$ \\
\hline & 13 & HDENS $\rightarrow \mathrm{G} 2$ \\
\hline Density & 14 & ISODENS $\rightarrow$ G1 \\
\hline & 15 & LDENS $\rightarrow \mathrm{G} 3$ \\
\hline & 16 & $\mathrm{C} 1 \rightarrow \mathrm{c} 1 \mid \mathrm{c} 1 . \mathrm{C} 1$ \\
\hline & 17 & $\mathrm{C} 2 \rightarrow \mathrm{c} 2 \mid \mathrm{c} 2 . \mathrm{C} 2$ \\
\hline & 18 & $\mathrm{C} 3 \rightarrow \mathrm{c} 3 \mid \mathrm{c} 3 . \mathrm{C} 3$ \\
\hline & 19 & $\mathrm{C} 4 \rightarrow \mathrm{c} 4 \mid \mathrm{c} 4 . \mathrm{C} 4$ \\
\hline & 20 & $\mathrm{~A} 1 \rightarrow \mathrm{a} 1 \mid \mathrm{a} 1 . \mathrm{A} 1$ \\
\hline & 21 & $\mathrm{~A} 2 \rightarrow \mathrm{a} 2 \mathrm{a} 2 . \mathrm{A} 2$ \\
\hline & 22 & $\mathrm{~B} 1 \rightarrow \mathrm{b} 1 \mid \mathrm{b} 1 . \mathrm{B} 1$ \\
\hline & 23 & $\mathrm{~B} 2 \rightarrow \mathrm{b} 2 \mid \mathrm{b} 2 . \mathrm{B} 2$ \\
\hline & 24 & $\mathrm{~B} 3 \rightarrow \mathrm{b} 3 \mid \mathrm{b} 3 . \mathrm{B} 3$ \\
\hline & 25 & $\mathrm{~B} 4 \rightarrow \mathrm{b} 4 \mid \mathrm{b} 4 . \mathrm{B} 4$ \\
\hline Elements & 26 & $\mathrm{~B} 5 \rightarrow \mathrm{b} 5 \mid \mathrm{b} 5 . \mathrm{B} 5$ \\
\hline of the & 27 & $\mathrm{~B} 6 \rightarrow \mathrm{b} 6 \mid \mathrm{b} 6 . \mathrm{B} 6$ \\
\hline diagnosed & 28 & $\mathrm{D} 1 \rightarrow \mathrm{d} 1 \mid \mathrm{d} 1 . \mathrm{D} 1$ \\
\hline lesion & 29 & $\mathrm{D} 2 \rightarrow \mathrm{d} 2 \mid \mathrm{d} 2 . \mathrm{D} 2$ \\
\hline & 30 & $\mathrm{D} 3 \rightarrow \mathrm{d} 3 \mid \mathrm{d} 3 . \mathrm{D} 3$ \\
\hline & 31 & 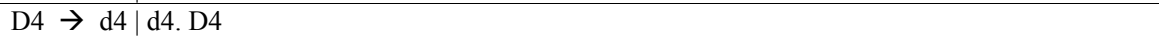 \\
\hline & 32 & $\mathrm{E} 1 \rightarrow \mathrm{e} 1 \mid \mathrm{e} 1 . \mathrm{E} 1$ \\
\hline & 33 & $\mathrm{E} 2 \rightarrow \mathrm{e} 2 \mid \mathrm{e} 2 . \mathrm{E} 2$ \\
\hline & 34 & $\mathrm{~F} 1 \rightarrow \mathrm{f} 1 \mid \mathrm{f} 1 . \mathrm{F} 1$ \\
\hline & 35 & $\mathrm{~F} 2 \rightarrow \mathrm{f} 2 \mid \mathrm{f} 2 . \mathrm{F} 2$ \\
\hline & 36 & $\mathrm{G} 1 \rightarrow \mathrm{g} 1 \mid \mathrm{g} 1 . \mathrm{G} 1$ \\
\hline & 37 & $\mathrm{G} 2 \rightarrow \mathrm{g} 2 \mid \mathrm{g} 2 . \mathrm{G} 2$ \\
\hline & 38 & $\mathrm{G} 3 \rightarrow \mathrm{g} 3 \mid \mathrm{g} 3 . \mathrm{G} 3$ \\
\hline
\end{tabular}

The ideal system yields a performance which is equal to $87.93 \%$. Moreover the FPR is $10.52 \%$. Therefore, using in-depth analyzed features and a cognitive classifier we have developed a trustable mammography CADx system. The system is trustable because its output comes with reason. In addition, the proposed cognitive classifier needs a low computational complexity because its major processes take place in the linguistics domain. Finally, the proposed cognitive CADx system yields a fair performance and FPR.

\section{CONCLUSION}

In this paper, a novel approach has been introduced for diagnosing masses in mammography images. The input ROI was subjected to some preprocessing stages such as histogram equalization, segmentation, and translation. Then, eleven in-depth analyzed features, which are proper descriptors of shape, margin, and density characteristics, have been extracted. The most effective features have been selected in the feature selection stage and mapped from the set of real numbers to a set of linguistics. The proposed classifier primes a knowledge base which has been developed according to a mammography expert's comments. The semantic comparison of features of real image with the expectations of knowledge base, which is called cognitive resonance, leads to the final assessment. Since the output of this system comes with reason, the system is trustable. The best achieved performance and FPR are $87.93 \%$ and $10.52 \%$, respectively. It was shown that the system is almost tolerable to the changes in the mapping intervals. Moreover, the proposed cognitive classifier needs a low computational complexity because its major processes take place in the linguistics domain.

Other researchers are advised to find a method which allows the plotting of ROC curves for such cognitive systems. For instance, a fuzzy rule base instead of a crisp one can be utilized in future works.

\section{REFERENCES}

[1] American Cancer Society, Breast Cancer Facts and Figures 2009-2010, Atlanta, 2009.

[2] C. A. Bovik, Handbook of Image and Video Processing, 2nd ed, Elsevier Academic Press, pp.1195 - 1217, 2005.

[3] A. Tahmasbi, F. Saki, and S. B. Shokouhi, "Classification of Benign and Malignant Masses Based on Zernike Moments," J. Computers in Biology and Medicine, vol. 41, no. 8, pp. 726-735, 2011.

[4] A. Tahmasbi, F. Saki, and S. B. Shokouhi, "An Effective Breast Mass Diagnosis System using Zernike Moments," in Proc. IEEE, 17th Iranian Conf. on Biomedical Engineering (ICBME'2010), Isfahan, Iran, pp. $1-4,2010$.

[5] A. Tahmasbi, F. Saki, and S. B. Shokouhi, "Mass Diagnosis in Mammography Images using Novel FTRD Features," in Proc. IEEE, 
17th Iranian Conf. on Biomedical Engineering (ICBME'2010), Isfahan, Iran, pp. 1-5, 2010.

[6] F. Saki, A. Tahmasbi, and S. B. Shokouhi, "A Novel Opposition-based Classifier for Mass Diagnosis in Mammography Images," in Proc. IEEE, 17th Iranian Conf. on Biomedical Engineering (ICBME'2010), Isfahan, Iran, pp. 1-4, 2010

[7] T. Z. Tan, C. Quek, G. S. Ng, and E. Y. K. Ng, "A novel cognitive interpretation of breast cancer thermography with complementary learning fuzzy neural memory structure," J Expert Systems with Applications, pp. 652-666, 2007.

[8] Q. Fang et al, "Combined Optical Imaging and Mammography of the Healthy Breast: Optical Contrast Derived from Breast Structure and Compression," IEEE Transactions on Medical Imaging, pp. 30 - 42, 2009.

[9] H. D. Cheng, X. J. Shi, R. Min, L. M. Hu, X. P. Cai, and H. N. Du, "Approaches for automated detection and classification of masses in mammograms,"J. Pattern Recognition, pp. 646 - 668, 2006.

[10] A. K. Jain, R.P.W. Duin, and J. Mao, "Statistical Pattern Recognition: A Review," IEEE Transactions on Pattern Analysis and Machine Intelligence, pp. $4-37,2000$.
[11] M. R. Ogiela, and R. Tadeusiewicz, "Nonlinear Processing and Semantic Content Analysis in Medical Imaging-A Cognitive Approach," IEEE Transactions on Instrumentation and Measurement, vol. 54 , no. 6 , pp. $2149-2155,2005$

[12] L. Ogiela, "Innovation Approach to Cognitive Medical Image Interpretation," in Proc. IEEE, International Conf. on Innovations in Information Technology (IIT), pp. 722 - 726, 2008.

[13] J. Suckling et al, "The Mammographic Image Analysis Society Digital Mammogram Database," J. Excerpta Medica, Int. Congress Series, pp. 375-378, 1994.

[14] W. Wang, J. E. Mottershead, and C. Mares, "Mode-shape recognition and finite element model updating using the Zernike moment descriptor," $J$ Mechanical Systems and Signal Processing, pp. 2088-2112, 2009

[15] L. M. Bruce, and R. R. Adhami, "Classifying Mammographic Mass Shapes Using the Wavelet Transform Modulus-Maxima Method," IEEE Transactions on Medical Imaging, pp. 1170 - 1177, 2009.

[16] A. R. Dominguez, and A. K. Nandi, "Development of Tolerant Features for Characterization of Masses in Mammograms," $J$ Computer in Biology and Medicine, 678-688, 2009. 